\title{
In-situ Pillared MXene as a viable Zinc-ion Hybrid Capacitor
}

\author{
Philip A Maughan ${ }^{1}$, Nuria Tapia-Ruiz ${ }^{2, *}$ and Nuno Bimbo ${ }^{1, *}$ \\ ${ }^{1}$ Department of Engineering, Lancaster University, LA1 $4 Y W$ \\ ${ }^{2}$ Department of Chemistry, Lancaster University, LA1 $4 Y B$
}

\begin{abstract}
MXenes are recently discovered two-dimensional materials which have shown great promise in electrochemical energy storage. However, the majority of research has been on lithium and sodium systems, with little work done on multivalent ion systems, which have an urgent need for new electrode materials. In particular, zinc-ion aqueous systems have significant promise due to the widespread use of zinc batteries and the abundance (24th most abundant element on earth), high specific capacity (> $800 \mathrm{mAh} \mathrm{g}^{-1}$ ) and low toxicity of zinc. Only a few materials are reported to act as reversible zinc-ion hosts, hindering developments of this technology. In this work, we demonstrate for the first time that $\mathrm{Ti}_{3} \mathrm{C}_{2}$, the most studied MXene to date, can act as a reversible zinc-ion host for a hybrid capacitor using an aqueous zinc sulphate electrolyte. In addition, we report a novel in-situ pillaring method where CTAB is used to increase the $\mathrm{Zn}$-ion uptake, with capacities up to $189 \mathrm{mAh} \mathrm{g}^{-1}$. A detailed mechanistic study that encompasses diffraction, microscopy and spectroscopy techniques was conducted to shed light on the structure and kinetics of the system. This understanding was used to optimise the electrode performance, resulting in an outstanding cycling stability of over $96 \%$ over 1,000 cycles. We believe this study will pave the pathway towards designing new pillared MXenes in low-cost $\mathrm{Zn}$-ion aqueous systems.
\end{abstract}

\section{Introduction}

There has been growing interest in electrochemical energy storage in recent years, with lithium-ion batteries going from powering consumer electronics to electric vehicles and grid-level energy storage.$^{1-3}$ However, lithium is a scarce and expensive element, which raises questions about its future sustainability, especially as demand continues to grow. ${ }^{4}$ In addition, there are wide-spread safety concerns about lithium-ion batteries, with worries about dendrite formation on the negative electrode (which can puncture the separator and cause a short circuit) and the flammability of the organic electrolyte used. ${ }^{5}$ These safety concerns are especially evident at high rates, which are needed to alleviate technological challenges associated with these applications. Consequently, there is an increasing focus on safer alternative metal-ion systems based on cations which are cheaper and more evenly distributed on earth. In particular, zinc systems are gaining significant interest. ${ }^{6,7}$ Zinc is relatively wide-spread in nature, has a large theoretical capacity $\left(>800 \mathrm{mAh} \mathrm{g}^{-1}\right)$ and a low potential (0.76 vs. standard hydrogen electrode (SHE)). ${ }^{8,9}$ Importantly, this potential sits in the stable voltage window for water, making zinc metal compatible with aqueous electrolytes, unlike most elements under consideration for rechargeable energy storage systems. ${ }^{10}$ Aqueous electrolytes are of interest since they are inherently non-flammable, which gives them significant safety advantages over systems based on organic electrolytes. However, aqueous electrolytes have significantly reduced voltage windows compared to organic electrolytes, due to the hydrogen and oxygen evolution reactions. ${ }^{11,12}$ This gives a thermodynamically stable window of $1.23 \mathrm{~V}$, which limits considerably the energy density of aqueous systems and makes the need to find high energy density electrode materials an urgent priority for these systems. 
Zinc is already widely used in primary (non-rechargeable) batteries, but commercial rechargeable systems have not yet been realised. This is in part because zinc metal forms dendrites when used with certain electrolytes, making the systems non-rechargeable in the long-term. However, it is now known that in some neutral and mildly acidic aqueous electrolytes (pH 4 to 6$)$, such as $1 \mathrm{M}$ zinc sulphate $\left(\mathrm{ZnSO}_{4}\right)$ in water, zinc can be reversibly cycled for long duration times without losing significant capacity. ${ }^{6}$

The majority of electrode materials belong to the oxide family and are based on manganese $\left(\mathrm{Mn}^{4+} /\right.$ $\left.\mathrm{Mn}^{2+}\right)^{13}$ and vanadium (e.g. $\mathrm{V}^{5+} / \mathrm{V}^{4+}$ in $\left.\mathrm{V}_{2} \mathrm{O}_{5}\right)^{14}$ redox couples, which store charge based on intercalation mechanisms. However, despite having relatively high capacities, typically between 100-350 mAh g-1, manganese oxides tend to suffer from poor cycling stability due to manganese dissolution in the electrolyte. ${ }^{15-17}$ Vanadium oxides generally have better cycling stability, but lower voltages than manganese-based ones. The capacities are typically in the range of $170-300 \mathrm{mAh} \mathrm{g}{ }^{-1} \cdot{ }^{18-20}$ In addition, Prussian blue analogues have recently been studied as a new class of zinc-ion electrode, but have shown poor capacities $\left(50-65 \mathrm{mAh} \mathrm{g}^{-1}\right)$ despite reasonably high operating voltages. ${ }^{21-23}$ So far, none of these materials classes have been able to meet the demands of a commercial zinc-ion energy storage system. ${ }^{7}$ Therefore, there is an urgent need for widespread research into new $\mathrm{Zn}^{2+}$ hosts with stable and reversible electrochemical performance. One difficulty has been that the smaller $\mathrm{Zn}$ ion size $(0.67$ $\AA ̊)$ and $2+$ charge give rise to poor diffusion kinetics, which has limited the range of available materials. In addition, the materials need to contain multivalent transition metals to allow them to be redox active with $\mathrm{Zn}$. One group of materials which have received lots of attention in other ion systems have been two-dimensional (2D) materials such as graphene. ${ }^{24-27}$ These are considered promising electrode materials due to the potential for high surface areas, large open structures between nanosheets, and high electrical conductivity which can result in hybrid capacitor mechanisms giving a combination of high energy densities, high rates and long cycle life. However, there have only been limited reports on the use of $2 \mathrm{D}$ materials in zinc-ion systems so far, with the work at a very early stage..$^{10,28,29}$

Another group of 2D materials are the recently discovered MXenes. ${ }^{30}$ These are a family of 2D transition metal carbides and nitrides synthesised from parent MAX phases (a family of layered ternary carbides where $M$ is an early transition metal, $A$ is an element from groups $13-16$ and $X$ is $C$ or $N$ ) through the selective removal of the $\mathrm{A}$ layer (e.g. Al), as demonstrated in Figure $1 \mathrm{a} .{ }^{31-33}$ While these have shown great early promise in a variety of different ion systems, including monovalent $\mathrm{Li}^{34-36}$ and $\mathrm{Na},{ }^{37-39}$ multivalent $\mathrm{Mg}^{40,41}$ and $\mathrm{Al}^{42}$ and aqueous supercapacitors, ${ }^{43-45}$ there has been limited reports on their performance in zinc-ion systems. ${ }^{46}$ To our knowledge, the only report of MXenes in Zn-ion is a very recent report by Yang et al., in which $\mathrm{Ti}_{3} \mathrm{C}_{2}$ MXene is used as an anode current collector to support zinc nanosheets and as an active cathode material via the electrostatic adsorption of $\mathrm{SO}_{4}{ }^{2-}$ ions. ${ }^{46}$ Wang et al. have also recently reported use of $\mathrm{Ti}_{3} \mathrm{C}_{2}$ as a positive electrode using anion adsorption. ${ }^{47}$ However, neither study demonstrates reversible $\mathrm{Zn}$-ion storage in an MXene. By far the most studied MXene is $\mathrm{Ti}_{3} \mathrm{C}_{2}$, which can be synthesised from commercially available MAX phases and has high electrical conductivity. ${ }^{48} \mathrm{Ti}_{3} \mathrm{C}_{2}$-based electrodes have been shown to be stable in aqueous supercapacitors over 1,000's of cycles, highlighting their compatibility with aqueous energy storage devices ${ }^{49,50}$, although $\mathrm{Ti}_{3} \mathrm{C}_{2}$ nanosheets can oxidise when stored in aqueous suspensions exposed to $\mathrm{air}^{51}$. Therefore, in this work, we have used $\mathrm{Ti}_{3} \mathrm{C}_{2}$ as a proof-of-concept system to study the electrochemical viability of MXenes as $\mathrm{Zn}$-ion hosts. Here, we demonstrate for the first time that $\mathrm{Ti}_{3} \mathrm{C}_{2}$ is active towards the insertion/removal of $\mathrm{Zn}$ ions, showing high reversible capacities and good capacity retention upon cycling. In addition, we present a simple, one-step in-situ pillaring approach 
in which a surfactant pillar (CTAB) is dissolved in the electrolyte and intercalated into the MXene to allow for high capacities in $\mathrm{Zn}$ storage by enabling a stable interlayer spacing. To the best of the authors' knowledge, this is the first time that this type of in-situ pillaring approach has successfully been used in MXenes. This work is expected to stimulate further research in this area by expanding the study to other MXene compositions as $\mathrm{Zn}^{2+}$ hosts and by seeking customised pillaring methods driven by structural and microstructural features of the MXene material and intercalation host.

\section{Experimental section}

\section{Synthesis of $\mathrm{Ti}_{3} \mathrm{C}_{2} \mathrm{~T}_{\mathrm{x}}$}

$3 \mathrm{~g}$ of $\mathrm{Ti}_{3} \mathrm{AlC}_{2}$ (Y-Carbon Ltd., Ukraine, 95\% purity) were sieved through a -400 mesh sieve (pore size of $38 \mu \mathrm{m}$ ) and added to a $6 \mathrm{M} \mathrm{HCl}$ solution (37.5\% wt. Sigma Aldrich) with pre-dissolved $\mathrm{NaF}$ (99\% purity, Alfa Aesar, 7.5:1 (F:Al molar ratio)) over $10 \mathrm{~min}$. The mixture was heated to $40^{\circ} \mathrm{C}$ and left to etch for $48 \mathrm{~h}$ under magnetic stirring. The solution was then centrifuged and the supernatant discarded. The sediments were re-dispersed in DI water and centrifuged for five further washing cycles until pH $=6$. The recovered $\mathrm{Ti}_{3} \mathrm{C}_{2}$ was then washed in $1 \mathrm{M} \mathrm{HCl}$ at room temperature for $3 \mathrm{~h}$ to remove any remaining salts. The MXene flakes were then dried overnight at $60{ }^{\circ} \mathrm{C}$.

\section{CTAB ex-situ pillared $\mathrm{Ti}_{3} \mathrm{C}_{2}$}

For hexadecyltrimethylammonium bromide (CTAB, Sigma Aldrich, >99\% purity) intercalation, $200 \mathrm{mg}$ of $\mathrm{Ti}_{3} \mathrm{C}_{2}$ were dispersed in a solution of DI water $(30 \mathrm{ml})$ and CTAB $(100 \mathrm{mg})$. The dispersion was stirred for $24 \mathrm{~h}$ at $40{ }^{\circ} \mathrm{C}$. The pillared MXene was then recovered by vacuum filtration and dried at $60^{\circ} \mathrm{C}$ overnight.

\section{Characterisation}

Powder X-ray diffraction. The samples were characterised by powder X-ray diffraction (PXRD) using a Smartlab diffractometer (Rigaku Corporation, Tokyo, Japan) with a $9 \mathrm{~kW} \mathrm{Cu}$ rotating anode operating in reflection mode with Bragg-Brentano geometry. Data were collected between 5 and $50^{\circ}$ $2 \theta$ at a scan rate of $1^{\circ} \mathrm{min}^{-1}$ (45 min). Prior to the PXRD characterisation, all samples were dried at $80^{\circ} \mathrm{C}$ for $18 \mathrm{~h}$, and the powders were ground with mortar and pestle and pressed flat onto a glass sample holder.

For ex-situ PXRD studies, the wet electrodes were taken immediately after disassembling the cell to the diffractometer, where they were placed flat on a metal support for data collection. This allowed for the interlayer spacing to be studied in a state close to that in the cell, whereas drying led to the removal of interlayer water, which is shown to play a key role in the structure of $\mathrm{Ti}_{3} \mathrm{C}_{2}$ during cycling. Data were collected between 5 and $50^{\circ} 2 \theta$ at a scan rate of $0.7^{\circ} \min ^{-1}(1 \mathrm{~h})$. For samples stopped at the open circuit voltage (OCV), the cell had been constructed and left to equilibrate for $24 \mathrm{~h}$ prior to disassembly and characterisation.

Raman spectroscopy. Raman spectroscopy was carried out on a Horiba Lab Raman Spectrometer (Horiba Ltd., Minamiku Kyoto, Japan) with an EM-cooled Synapse camera. For taking spectra, a 100x, 0.90 NA microscope objective was used. The dried electrodes were placed with the MXene side up under the diode laser $(532 \mathrm{~nm}, 200 \mu \mathrm{W})$ for measurements.

Scanning electron microscopy. Scanning electron microscopy (SEM) was performed in a JEOL JSM$7800 \mathrm{~F}$ (JEOL Ltd., Tokyo, Japan), and energy-dispersive x-ray spectroscopy (EDS) was performed in an $\mathrm{X}$-Max50 (Oxford Instruments plc, Abingdon, UK) using an accelerating voltage of $10 \mathrm{kV}$ and a working distance of $10 \mathrm{~mm}$ to study sample morphology and elemental composition. For the SEM and EDS 
studies, the dried powder samples were dry cast onto a carbon tape support, which was placed onto a copper stub for analysis.

X-ray photoelectron spectroscopy. X-ray photoelectron spectroscopy (XPS) was performed on a Thermo Fisher Scientific NEXSA spectrometer. Samples were analysed using a micro-focused monochromatic Al x-ray source (19.2 W) over an area of approximately 100 microns. Data were recorded at pass energies of $150 \mathrm{eV}$ for survey scans and $40 \mathrm{eV}$ for high resolution scan with $1 \mathrm{eV}$ and $0.1 \mathrm{eV}$ step sizes respectively. Charge neutralisation of the sample was achieved using a combination of both low energy electrons and argon ions. To remove any surface contaminants, cluster cleaning was performed with $2 \mathrm{keV}$ energy at $0.5 \times 0.5 \mathrm{~mm}$ area for $60 \mathrm{~s}$. The electrodes were extracted from the cell at the desired state of charge, washed 5 times with DI water to remove surface salts, dried at room temperature under vacuum, and packed under argon before testing.

Electrochemical characterisation. The electrochemical performance of the MXene materials in aqueous $\mathrm{Zn}$-ion hybrid capacitors was tested in stainless steel CR2032 coin cells using zinc foil (Tob New Energy) disks as the counter and reference electrode, $0.1 \mathrm{M} \mathrm{ZnSO}_{4}$ (zinc sulphate heptahydrate, $>99 \%$, Sigma Aldrich) in DI water as the electrolyte and a Whatman micro glass fibre separator. For the in-situ pillaring experiments, $0.1 \mathrm{wt} . \%$ of CTAB was added to this electrolyte. The MXene electrode had an active material: carbon black (Super P, 99\% purity, Alfa Aesar): PVDF (99.0\% purity, Alfa Aesar) mass ratio of 75:15:10. N-Methyl-2-pyrrolidone (NMP, 99.5\%, Alfa Aesar, $1.7 \mathrm{dm}^{-3}$ ) was added to the mixture to make a slurry, which was then cast onto a titanium foil (Tob New Energy, $100 \mu \mathrm{m}$ ) current collector, from which electrodes with a diameter of $16 \mathrm{~mm}$ were punched. The active mass weighting was around $2.8 \mathrm{mg} \mathrm{cm}^{-2}$. Galvanostatic measurements were carried out on a Neware battery cycler (Neware Technology Ltd, China) at room temperature. All potentials are reported with respect to $\mathrm{Zn}^{2+} / \mathrm{Zn}$. The charge/discharge tests were carried out at a current density of $20 \mathrm{~mA} \mathrm{~g}^{-1}$ in the voltage range of 0.01-1 V. For rate capability tests, the cells were cycled at a current density of $20 \mathrm{~mA} \mathrm{~g}^{-1}$ for 1 cycle to stabilise the cell before 5 cycles at each current density of 20,50, 200, 500 and 1,000 mA g-

${ }^{1}$ were run before returning to $20 \mathrm{~mA} \mathrm{~g}^{-1}$. Cyclic voltammetry measurements were conducted using an Ivium potentiostat (Ivium Technologies BV, The Netherlands) with a scan rate of $0.1 \mathrm{mV} \mathrm{s}^{-1}$ for 2 cycles, followed by 2 further cycles each at $0.5,2$ and $5 \mathrm{mV} \mathrm{s}^{-1}$ sweep rates in the voltage range of 0.01-1V.

\section{Results and Discussion}

\section{Structural Characterisation}

PXRD data shows that the MXene was successfully etched from the parent MAX phase (Figure 1c). This is indicated by the loss of typical $\mathrm{Ti}_{3} \mathrm{AlC}_{2}$ diffraction peaks ${ }^{30,44}$ and the shift of the (002) peak to lower angles (from 9.6 to $8.1^{\circ} 2 \theta$ ), which corresponds to the increased interlayer spacing after the removal of the Al layer. SEM data confirm that the layered structure of the MXene is retained after etching (Figure 1b), in agreement with previous reports. ${ }^{30,44}$ EDS results show negligible Al content in the etched MXene ( 0.2 wt.\%), confirming the transformation from $\mathrm{Ti}_{3} \mathrm{AlC}_{2}$ to $\mathrm{Ti}_{3} \mathrm{C}_{2}$ (Figure S1). EDS results also show that the $\mathrm{Ti}_{3} \mathrm{C}_{2}$ contains the common terminal elements $\mathrm{O}(11 \mathrm{wt} . \%), \mathrm{F}(6 \mathrm{wt} . \%)$ and $\mathrm{Cl}(2.5$ wt.\%) which bind to the Ti layer during the etching process. The XRD data for the in-situ pillared sample is shown in blue (Figure 1c). We observed an increase in the interlayer spacing in the material through a further shift in the (002) diffraction peak from 8.1 to $5.9^{\circ} 2 \theta$, which confirms that successful pillaring took place. This corresponds to an increase in d-spacing from $1.1 \mathrm{~nm}$ in the as-etched MXene to 1.5 $\mathrm{nm}$ in the in-situ pillared material. A single $\mathrm{Ti}_{3} \mathrm{C}_{2}$ layer has a reported thickness of $0.95 \mathrm{~nm},{ }^{52}$ therefore, we estimate that the pore space between the layers has experienced a 3.7-fold increase (i.e. from 0.15 
$\mathrm{nm}$ to $0.55 \mathrm{~nm}$ ) after using the reported pillaring method. For comparison, we used a traditional exsitu pillared approach in $\mathrm{Ti}_{3} \mathrm{C}_{2}$ (Figure $\mathrm{S} 2$ ), which is consistent with the interlayer spacing values obtained from the $\mathrm{Ti}_{3} \mathrm{C}_{2}$ material pillared using the in-situ method. Previous reports of pillaring with $C T A B$ have used time-consuming and highly parameter sensitive methods which give a range of interlayer spacings demonstrating that these pre-pillaring techniques have several drawbacks. ${ }^{40,52} \mathrm{It}$ is therefore significant that we can obtain the same pillaring effect with such a simple in-situ method, which hugely simplifies the manufacturing process for pillared MXenes.

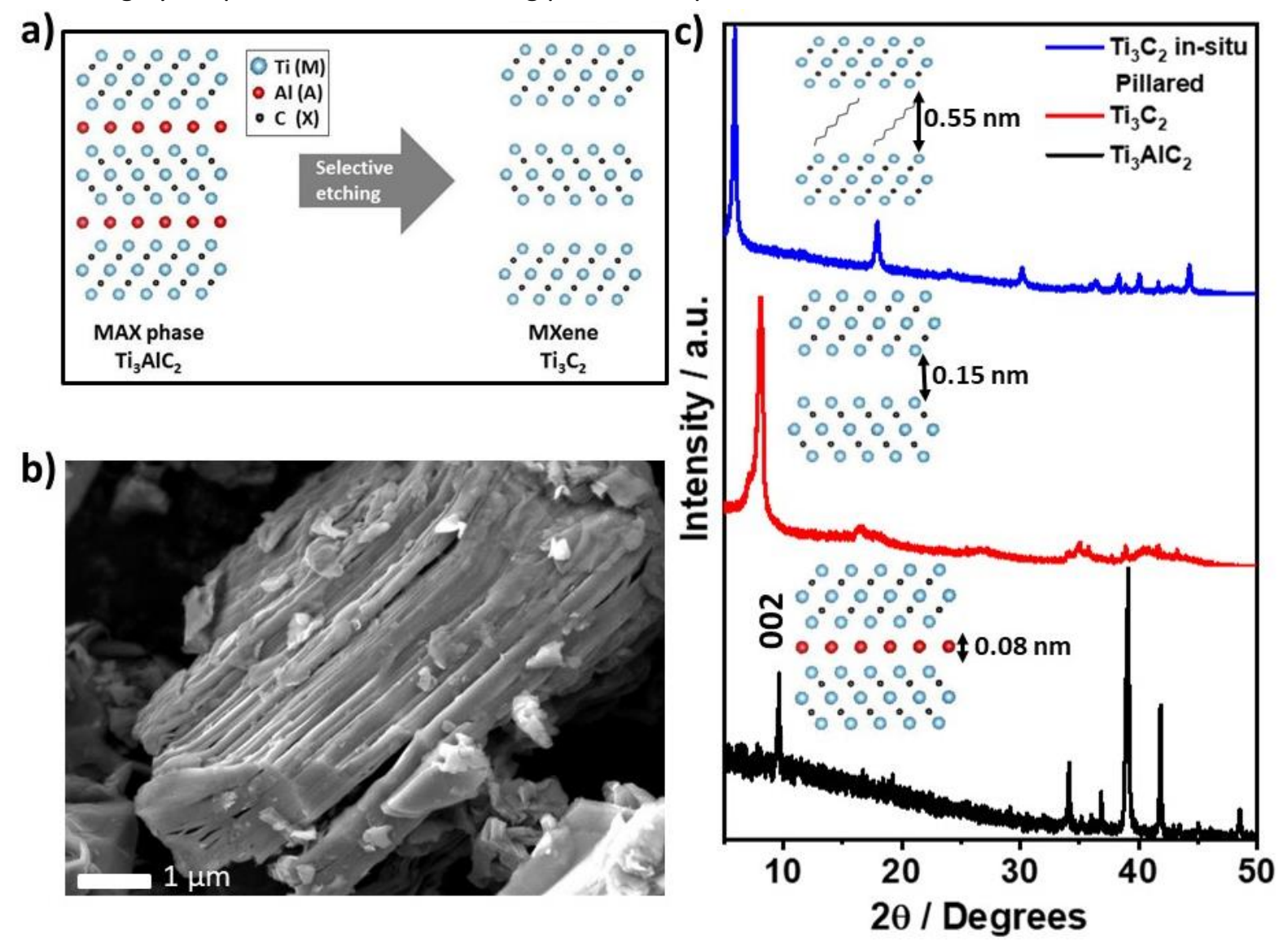

Figure 1. a) Schematic of the synthesis of $\mathrm{Ti}_{3} \mathrm{C}_{2}$ via selective etching of $A$ l from the $\mathrm{Ti}_{3} \mathrm{AlC}_{2} \mathrm{MAX}$ phase precursor. b) SEM image of as-synthesised $\mathrm{Ti}_{3} \mathrm{C}_{2} \mathrm{MXene}$ flake showing the typical layered morphology of MXenes. c) XRD of the commercial $\mathrm{Ti}_{3} \mathrm{AlC}_{2}$ precursor MAX phase (black), as-etched $\mathrm{Ti}_{3} \mathrm{C}_{2}$ (red) and in-situ pillared (blue) after drying at $60^{\circ} \mathrm{C}$ for $18 \mathrm{~h}$. The (002) diffraction peak (labelled) relates directly to the interlayer spacing of these materials and shows substantial shifts both after etching and pillaring. The insets illustrate the change in the interlayer distance across these samples.

\section{Electrochemical characterisation}

Galvanostatic charge/discharge tests were carried out on the in-situ pillared MXene in the voltage window of 0.01-1 $\mathrm{V}$ using a current density of $20 \mathrm{~mA} \mathrm{~g}^{-1}$ (Figure 2). Figure 2a contains a schematic illustrating the in-situ pillaring process whereby $\mathrm{CTA}^{+}$is intercalated between the $\mathrm{Ti}_{3} \mathrm{C}_{2}$ layers on the first discharge cycle inside the $\mathrm{Zn}$-ion hybrid capacitor. The material shows a significant reversible $\mathrm{Zn}$ ion uptake/removal behaviour and a sloping voltage profile, which is consistent with a capacitive-type charge storage mechanism with no major phase changes during cycling. This is in agreement with data reported for $\mathrm{Ti}_{3} \mathrm{C}_{2}$ in other metal-ion systems. ${ }^{34,37,53,54}$ Furthermore, ex-situ XRD results show no phase changes occur during cycling, as is described in a later section. An initial discharge capacity of $189 \mathrm{mAh}$ $\mathrm{g}^{-1}$ was achieved followed by a second cycle discharge capacity of $92 \mathrm{mAh} \mathrm{g}^{-1}$. This initial irreversible 
capacity loss is further investigated by ex-situ Raman spectroscopy in a later section. After the second cycle, a capacity retention of $70 \%$ was achieved over 20 cycles (Figure $2 \mathrm{~d}$ ). An initial low coulombic efficiency was observed in the first cycle (39\%), which is analogous to that observed in organic Li and Na systems. ${ }^{37,53}$ This has been explained by the formation of a solid-electrolyte interphase (SEI) and trapping of the charge carrying cation by an irreversible reaction with some surface groups on the MXene. For comparison, we also tested the electrochemical behaviour (under the same conditions) of the as-made MXene and pre-pillared sample and observed lower initial discharge capacities (108 and $105 \mathrm{mAh} \mathrm{g}^{-1}$ ), respectively, when compared to the in-situ pillared MXene (Figure S3). The asprepared and ex-situ pillared MXene showed larger initial coulombic efficiencies (52\% and 69\%, respectively) when compared to the in-situ pillared MXene (39\%). We explain these differences with the greater exposure to the electrolyte of reactive sites in the in-situ pillared MXene, leading to greater SEl formation and $\mathrm{Zn}$ trapping.
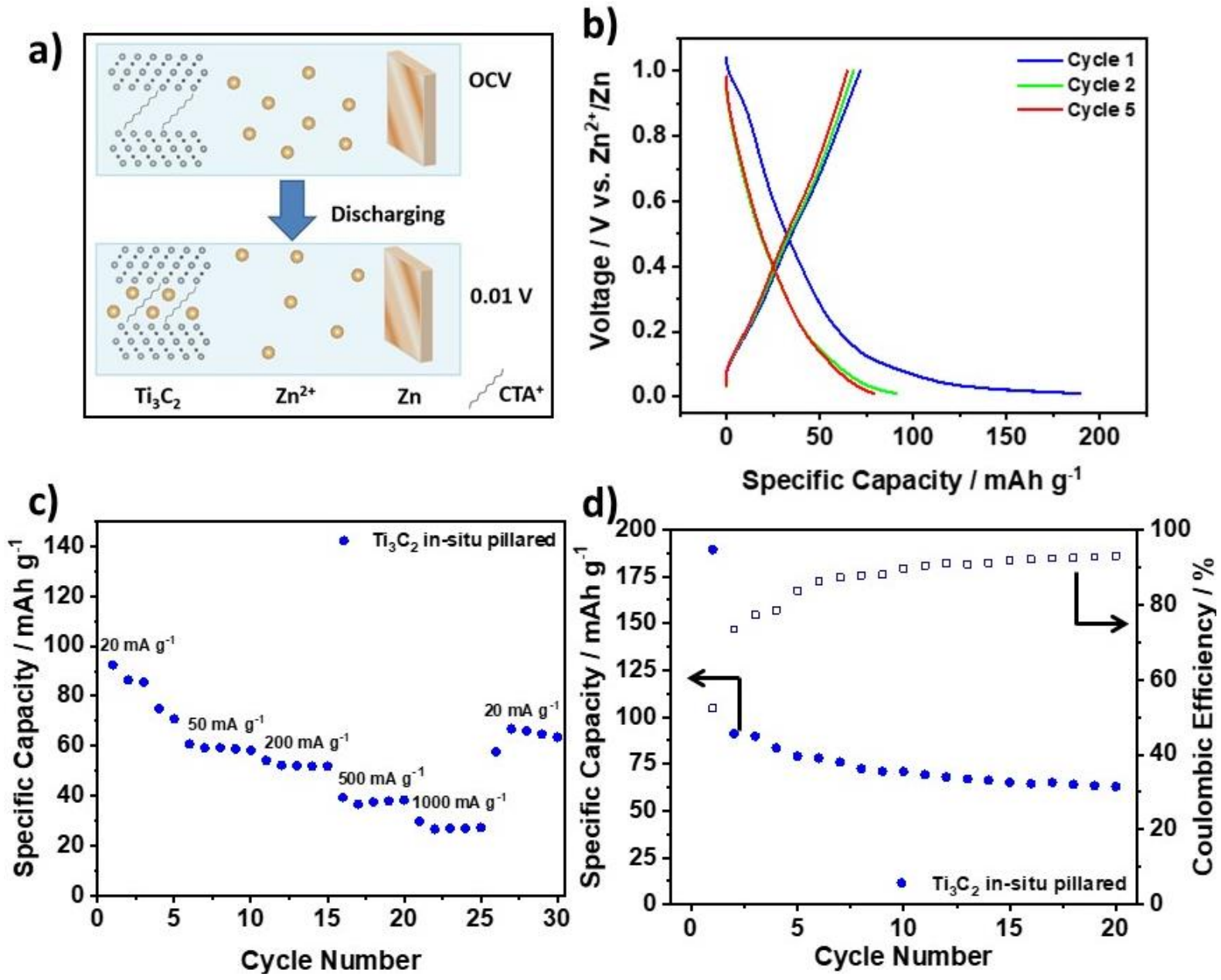

Figure 2. a) Schematic of the in-situ pillaring process. (b) Load curves in the voltage window of 0.01-1 $V$ at a current density of $20 \mathrm{~mA} \mathrm{~g}^{-1}$ of the in-situ pillared $\mathrm{Ti}_{3} \mathrm{C}_{2}$. (c) Rate capability tests for in-situ pillared $\mathrm{Ti}_{3} \mathrm{C}_{2}$ at rates of 20,50,200, 500 and $1,000 \mathrm{~mA} \mathrm{~g}^{-1}$; and (d) Discharge capacities and Coulombic efficiencies over 20 cycles at a rate of $20 \mathrm{~mA} \mathrm{~g}^{-1}$ across a voltage window of $0.01-1 \mathrm{~V}$ for in-situ pillared $\mathrm{Ti}_{3} \mathrm{C}_{2}$.

Rate capability (Figure 2c) and stability (Figure 2d) tests were also carried out in the in-situ pillared MXene to further investigate the electrochemical behaviour of $\mathrm{Ti}_{3} \mathrm{C}_{2}$ in the $\mathrm{Zn}$-ion system. The in-situ pillared MXene also performed well under rate capability testing when cycled at increasing rates of at 
$20,50,200,500$ and $1,000 \mathrm{~mA} \mathrm{~g}^{-1}$. The in-situ pillared material has the highest capacity at each rate, achieving average capacities of $86 \mathrm{mAh} \mathrm{g}^{-1}, 59 \mathrm{mAh} \mathrm{g}^{-1}, 52 \mathrm{mAh} \mathrm{g}^{-1}, 38 \mathrm{mAh} \mathrm{g}^{-1}$ and $27 \mathrm{mAh} \mathrm{g}^{-1}$ at 20, $50,200,500$ and $1,000 \mathrm{~mA} \mathrm{~g}^{-1}$ respectively. After the high rate testing, the rate was switched back to $20 \mathrm{mAh} \mathrm{g}^{-1}$ and the capacity recovered back to $67 \mathrm{mAh} \mathrm{g}^{-1}$. This shows that the in-situ pillared MXene responds well to high rate cycling and is not damaged by fast charging or discharging. The in-situ pillared MXene retained a discharge capacity of $62 \mathrm{mAh} \mathrm{g}^{-1}$ after 20 cycles $\left(70 \%\right.$ of the $2^{\text {nd }}$ cycle capacity). This loss is likely to be due to the degradation of the electrode and/or irreversible reactions with the charge carrier but is still less than the loss observed for the non-pillared and ex-situ pillared materials (Figure $2 \mathrm{~d}$ for the in-situ pillared $\mathrm{Ti}_{3} \mathrm{C}_{2}$ and Figure S3d for the non-pillared and ex-situ pillared $M X e n e)$. In comparison, the as-made MXene only had a capacity of $54 \mathrm{mAh} \mathrm{g}^{-1}$ after 20 cycles and the CTAB ex-situ pillared material showed only $42 \mathrm{mAh} \mathrm{g}^{-1}$. In the rate capability tests, the as-made MXene only showed capacities of $61,45,40,27$ and $17 \mathrm{mAh} \mathrm{g}^{-1}$ at the respective rates. When the rate was returned to $20 \mathrm{~mA} \mathrm{~g}^{-1}$, the capacity recovered to $56 \mathrm{mAh} \mathrm{g}^{-1}$, again showing that the electrode is not damaged by high rates.

Cyclic voltammetry (CV) studies were carried out on the in-situ pillared system to gain more insight into the $\mathrm{Zn}$ storage mechanism and the kinetics of the system (Figure 3 ). Cells were first cycled at a sweep rate of $0.1 \mathrm{mV} \mathrm{s}^{-1}$ for 2 cycles between 0.01 and $1 \mathrm{~V}$ before further cycling at sweep rates of $0.5,2$ and $5 \mathrm{mV} \mathrm{s}^{-1}$. The material shows a quasi-rectangular CV plot, which suggests that there is a large capacitive contribution to the storage mechanism in this material. However, there are broad reversible redox peaks at $0.3 \mathrm{~V}$ (with a shoulder at $0.2 \mathrm{~V}$ ) and at $0.7 \mathrm{~V}\left(\mathrm{vs} . \mathrm{Zn}^{2+} / \mathrm{Zn}\right.$ ) on the anodic sweep and at $0.11 \mathrm{~V}$ and $0.5 \mathrm{~V}$ on the cathodic sweep. An irreversible peak at ca. $0.1 \mathrm{~V}$ on the cathodic sweep was observed on the first cycle, which could be due to SEI formation. This is in contrast to the reported CV plots of $\mathrm{Ti}_{3} \mathrm{C}_{2}$ in other sulphate electrolytes such as $\mathrm{H}_{2} \mathrm{SO}_{4}, \mathrm{Li}_{2} \mathrm{SO}_{4}$ and $\mathrm{Na}_{2} \mathrm{SO}_{4},{ }^{43-45,55}$ where no sharp redox peaks are observed and the plots are much more rectangular in shape. This suggests that the $\mathrm{MXene}$ may behave differently in the aqueous $\mathrm{ZnSO}_{4}$-based electrolyte than it does in other sulphatebased electrolytes based on monovalent cations. When the sweep rate is increased, the $\mathrm{CV}$ plots retain the quasi-rectangular shape and the broad cathodic peaks at 0.2 and $0.7 \mathrm{~V}$ are still noticeable at all rates but have shifted to higher voltages as the rate increases (to 0.4 and $0.95 \mathrm{~V}$ respectively by $5 \mathrm{mV}$ $\left.\mathrm{s}^{-1}\right)$. Individual CV plots at different sweep rates are available in Figure S5. 

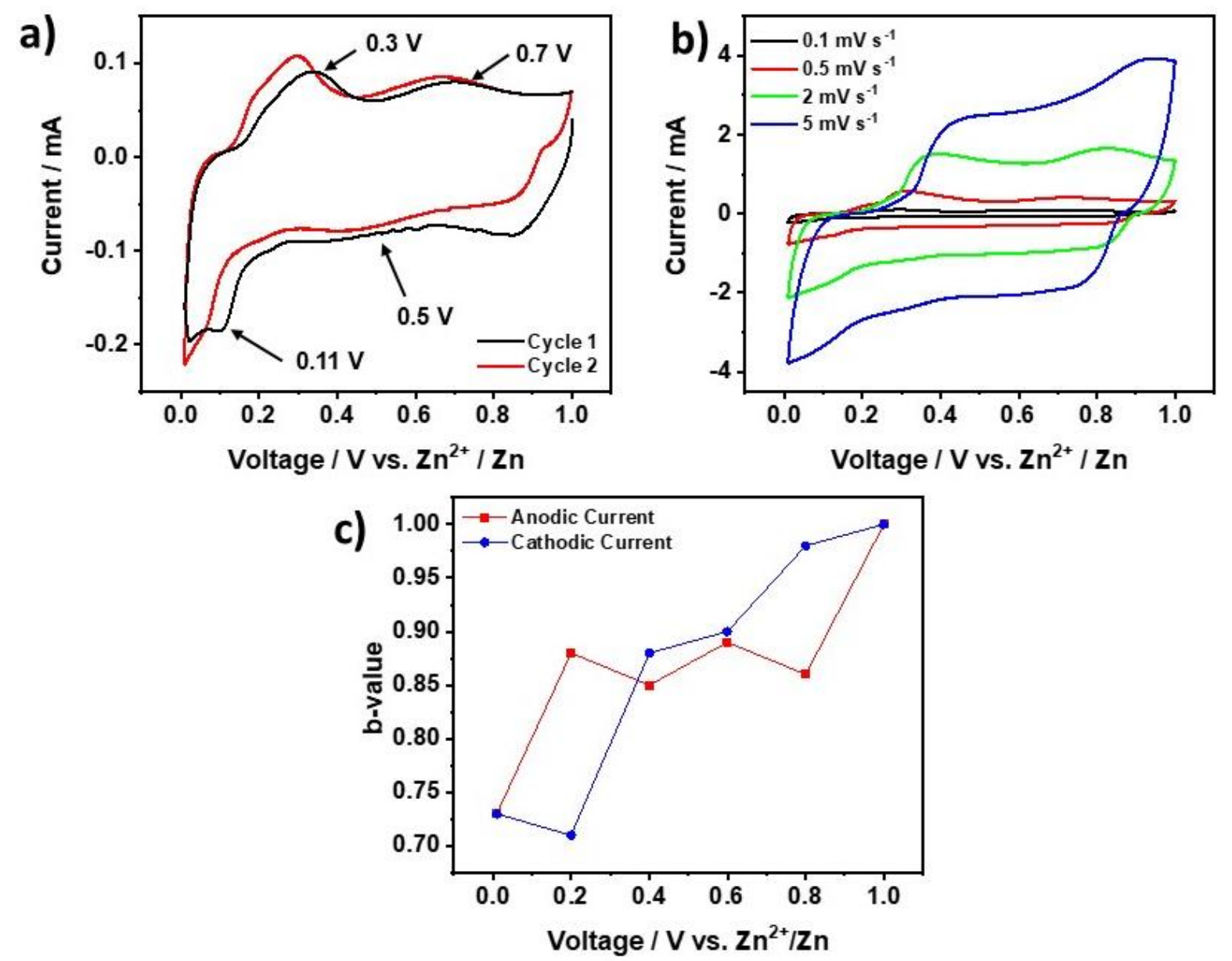

Figure 3. Cyclic voltammograms for: a) in-situ pillared $\mathrm{Ti}_{3} \mathrm{C}_{2}$ at $0.1 \mathrm{mV} \mathrm{s}^{-1}$ in a voltage window of 0.01 $1 \mathrm{~V}$ for 2 cycles b) CV plots for in-situ pillared $\mathrm{Ti}_{3} \mathrm{C}_{2}$ at $0.1,0.5,2$ and $5 \mathrm{mV} \mathrm{s}^{-1}$. ( $1^{\text {st }}$ cycle at each sweep rate is shown) c) Plot of b-value vs. voltage for the in-situ pillared $\mathrm{Ti}_{3} \mathrm{C}_{2}$ for the anodic (red) and cathodic (blue) sweeps.

There are 3 main charge storage mechanisms found in electrochemical energy storage devices: faradaic diffusion-limited redox reactions (e.g. intercalation reactions found in typical battery materials), faradaic surface-controlled redox reactions (i.e. pseudocapacitance) and non-faradaic electric double layer capacitance. It is known that the contribution of the diffusion-limited contribution and the capacitive contribution to the charge storage can be separated out using the CV profiles at different rates. ${ }^{56}$ The relationship between the current $(i)$ and scan rate $(v)$ is given in equation 1:

$$
i=a v^{b}
$$

where $a$ and $b$ are fitting parameters. ${ }^{57}$ Importantly, a $b$-value of 0.5 corresponds to a diffusion-limited process (i.e. battery-like charge storage) and a $b$-value of 1 indicates a surface-controlled process (i.e. capacitive charge storage). A plot of $\log (i)$ against $\log (v)$ gives a straight line with a slope of $b$. In this work, $b$-values of 0.85 and 0.84 were obtained for the in-situ pillared MXene, showing a great contribution from capacitive processes. This is in agreement with previous studies reported in the literature for this material in different ion systems, where $b$-values of $0.8-1$ are typical. When plotting the $b$-value obtained at different voltages during cathodic and anodic processes (Figure $3 c$ ), a 
significant voltage dependence for the mechanism of charge storage was observed. During discharge, a predominant capacitive process occurs, given by the high values of $b$ (which fall between 1 and 0.9), followed by a diffusion-limited process occurring $(<0.4 \mathrm{~V})$. These results match well the CV plots, where there are distinctive peaks in the voltage region of 0.2-0.4 V (Figure 3a). Upon charge, the proportion of the capacitive contribution to the charge storage mechanisms rapidly increases, with $b$ values of ca. 0.9 between 0.2 to $0.8 \mathrm{~V}$, with an increase to $b=1$ from $0.8 \mathrm{~V}$. This suggests that the anodic peaks observed in the $\mathrm{CV}$ plots have a minimal contribution to redox processes. Overall, these data confirm the complexity of the Zn storage mechanism occurring in the MXene, which behaves as a hybrid capacitor electrode, where low states of charge will favour diffusion processes whilst high states of charge will induce capacitive processes.

\section{Structural changes during cycling}

To study the structural changes during cycling, ex-situ PXRD studies were carried out on the in-situ pillared (Figure 4) and non-pillared (Figure S6) MXene. Figure 4 shows that the interlayer distance at $\mathrm{OCV}$ for the in-situ pillared $\mathrm{Ti}_{3} \mathrm{C}_{2}$ is significantly enlarged compared to the pristine MXene, with a shift of the (002) diffraction peak from $8.1^{\circ} 2 \theta$ in the pristine material to $5.9^{\circ} 2 \theta$ at OCV. This is a larger interlayer spacing than that reported for $\mathrm{Ti}_{3} \mathrm{C}_{2}$ in organic electrolytes for $\mathrm{Li}$ and $\mathrm{Na}$ systems, which typically see the peak shift to around $7^{\circ} 2 \theta$, which is only visible in our data as a small peak at OCV (starred, inset Figure 4). ${ }^{37,53}$ The (002) diffraction peak relates directly to the interlayer distance and pore height in the electrode material and here corresponds to a pore height of around $0.55 \mathrm{~nm}$ (interlayer spacing of $1.5 \mathrm{~nm}$ ), which is significantly larger than the ratio of a $\mathrm{Zn}^{2+}$ ion $(0.074 \mathrm{~nm}) \cdot{ }^{58} \mathrm{EDS}$ (Table S1) confirms that $\mathrm{Zn}$ is detected in the MXene electrode after being soaked in the electrolyte, showing that it can spontaneously intercalate into $\mathrm{Ti}_{3} \mathrm{C}_{2}$. Given that $\mathrm{Zn}$ has a hydrated ionic radius of $0.43 \mathrm{~nm}^{6}$ it is likely that this is intercalated in a solvated state $\left(\left[\mathrm{Zn}\left(\mathrm{H}_{2} \mathrm{O}\right)_{6}\right]^{2+}\right) \cdot{ }^{59}$ Large XRD shifts arising from the spontaneous intercalation of a variety of other cations into $\mathrm{Ti}_{3} \mathrm{C}_{2}$ has previously been reported. ${ }^{55}$ No significant difference is observed between the in-situ pillared and non-pillared MXene (Figure S6) at this stage. 


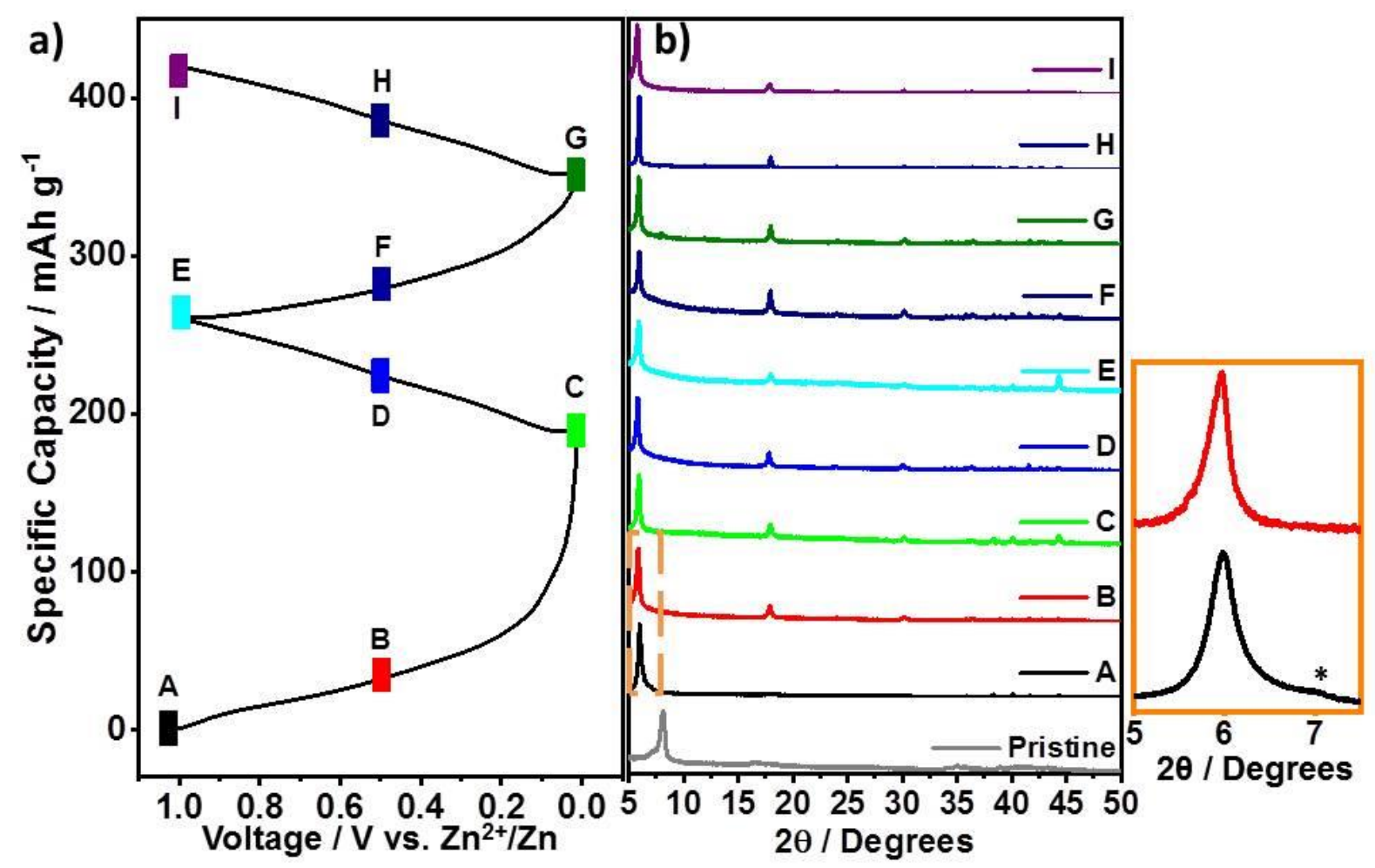

Figure 4. a) Ex-situ XRD data of the in-situ pillared $\mathrm{Ti}_{3} \mathrm{C}_{2}$ material at different states of charge in the voltage window 0.01-1 V. a) Load curve highlighting the state of charge at which each electrode was stopped at and analysed; b) Corresponding X-ray diffraction data for each state of charge. The orange rectangle shows a zoom in the region of the (002) diffraction peak at OCV state and during the first discharge to $0.5 \mathrm{~V}$, highlighting the loss of the shoulder (asterisk shows small shoulder peak at $7^{\circ} 2 \theta$ ) and sharpening of the peak as all layers appear to be intercalated.

Upon discharging to $0.5 \mathrm{~V}$, almost no change is detected in the XRD data compared to the material at $O C V$, apart from the evolution of two peaks at $18^{\circ}$ and $30^{\circ} 2 \theta$, which are assigned as the (004) and (006) diffraction peaks of $\mathrm{Ti}_{3} \mathrm{C}_{2}$ which implies that the insertion of $\mathrm{Zn}$ ions in the MXene enhances the layer ordering within the MXene crystal structure. The (002) diffraction peak at $5.9^{\circ} 2 \theta$ has sharpened slightly, suggesting a uniform increase of the interlayer distances. This implies that $\mathrm{Zn}$ has been intercalated into $\mathrm{Ti}_{3} \mathrm{C}_{2}$, which has expanded previously unfilled interlayers which made up the shoulder of the (002) diffraction peak including small starred peak and caused a slight shift to lower angles i.e. a larger interlayer distance (see the inset of Figure $4 \mathrm{~b}$ ). Over subsequent states-of-charge, no extra peaks from new phases are observed, confirming that the $\mathrm{Zn}$ storage occurs without a phase change, which fits well with the sloping profile of the load curve (Figure $2 b$ ). In addition, the (002) diffraction peak is at $5.9^{\circ} 2 \theta$ at all states of charge showing that the pillared electrode sees negligible volume changes during cycling. There is negligible variation in the position of this peak, which is likely to be due to small differences in dehydration of the samples for each run, which can be due to sample preparation and measurement. That the interlayer spacing remains constant despite the deintercalation of $\mathrm{Zn}^{2+}$ (as shown by the EDS results in Table S1) is explained by the intercalation of the $\mathrm{CTA}^{+}$pillars and/ or water molecules, which are not removed during the discharge sweeps, therefore allowing a stable interlayer distance during cycling. It has been previously reported that organic electrolyte solvent molecules can act as small pillars during cycling, where minimal decrease in the interlayer spacing upon deintercalation of $\mathrm{Na}^{+}$was observed. ${ }^{60}$ Therefore, these results highlight 
the successful use of $\mathrm{CTA}^{+}$pillars to create an MXene electrode with negligible volume change during cycling.

For the non-pillared MXene, a new peak at $8.4^{\circ} 2 \theta$ is observed when the electrode is fully discharged to $0.01 \mathrm{~V}$ (Figure S6). This is assigned as a split of the (002) diffraction peak with a smaller interlayer distance than the $5.9^{\circ} 2 \theta$ peak. No other change is observed, confirming that $\mathrm{Zn}$ storage occurs without a phase change. When the cell is charged to $1 \mathrm{~V}$, the diffraction peak at $8.4^{\circ} 2 \theta$ disappears, showing that all the MXene sheets are evenly spaced again. The same pattern is repeated on the $2^{\text {nd }}$ cycle.

In an ex-situ study of $\mathrm{Ti}_{3} \mathrm{C}_{2}$ in an organic lithium electrolyte, Cheng et al. also found that the (002) diffraction peak split and that the intensity of the high angle peak increased at low voltages (discharged state) and decreased in intensity when the voltage increased (charged state). ${ }^{53}$ They assigned this to a sequential intercalation mechanism where the $\mathrm{Li}$ intercalated into $\mathrm{Ti}_{3} \mathrm{C}_{2}$ with the large interlayer spaced sites preferentially (i.e. first) at the higher voltages. An alternative explanation could be that at low voltages (as the cell discharges and extra $\mathrm{Zn}$ intercalates), some solvent (water) molecules are expelled from the interlayer space, potentially allowing $\mathrm{Zn}^{2+}$ to pull the layers together through electrostatic attraction. This process of pulling the sheets together and expelling water or intercalation of $\mathrm{Zn}^{2+}$ into smaller sites would correspond to the faradaic process identified via the CV analysis at low voltages (Figure 3).

By contrast, the peak split is not visible for the in-situ pillared material. Cheng et al. also noticed that when $\mathrm{NH}_{4}{ }^{+}$was pre-intercalated into $\mathrm{Ti}_{3} \mathrm{C}_{2}$, the interlayer spacing increased, removing the sites with small interlayer spacings. ${ }^{53}$ Here we observe the same effect with the in-situ pillaring method which suggests that $\mathrm{CTA}^{+}$was successfully intercalated between the layers and it is indeed acting as an insitu pillar for the MXene. Furthermore, the pillar also increases the accessibly of the surface to the solvated $\mathrm{Zn}^{2+}$ ions, which improves the capacity, since more storage sites are available (Figures 2 and S3). The rate capability also improves due to reduced interaction between the $\mathrm{Zn}$ ions and the MXene surface before reaching a storage site (Figures $2 \mathrm{c}$ and $\mathrm{S} 3 \mathrm{c}$ ). This demonstrates a low diffusion barrier, explaining the high proportion of current derived via capacitive surface-controlled processes (Figure 3).

Raman spectroscopy has previously been demonstrated to be a useful tool in studying the nature of MXene surface groups since their Raman spectra are highly sensitive to changes in the surface chemistry. ${ }^{45,61}$ Consequently, it can give key insights into the role of MXene functional groups and the charge storage mechanism. ${ }^{52}$ Figure 5 shows the ex-situ Raman spectra of the in-situ pillared MXene in the first charge-discharge cycle (the corresponding data for the non-pillared $\mathrm{Ti}_{3} \mathrm{C}_{2}$ can be found in Figure S7). For comparison, the Raman spectrum for the pristine cast electrode is also included. There are common peaks in all spectra, which are consistent with those previously reported for $\mathrm{Ti}_{3} \mathrm{C}_{2}{ }^{45,61}$ The peaks at around $210 \mathrm{~cm}^{-1}$ and $740 \mathrm{~cm}^{-1}$ correspond to vibrations from Ti=O terminated MXene, whereas the broad peak at $270 \mathrm{~cm}^{-1}$ results from the presence of Ti-O-H groups (i.e. protonated oxygen terminations). The large and very broad peaks located between 350 and $450 \mathrm{~cm}^{-1}$ and 550 and 700 $\mathrm{cm}^{-1}$ correspond to vibrations from all the surface groups present in the MXene, which are known to have significant overlap in their vibrational frequencies. ${ }^{61}$ 

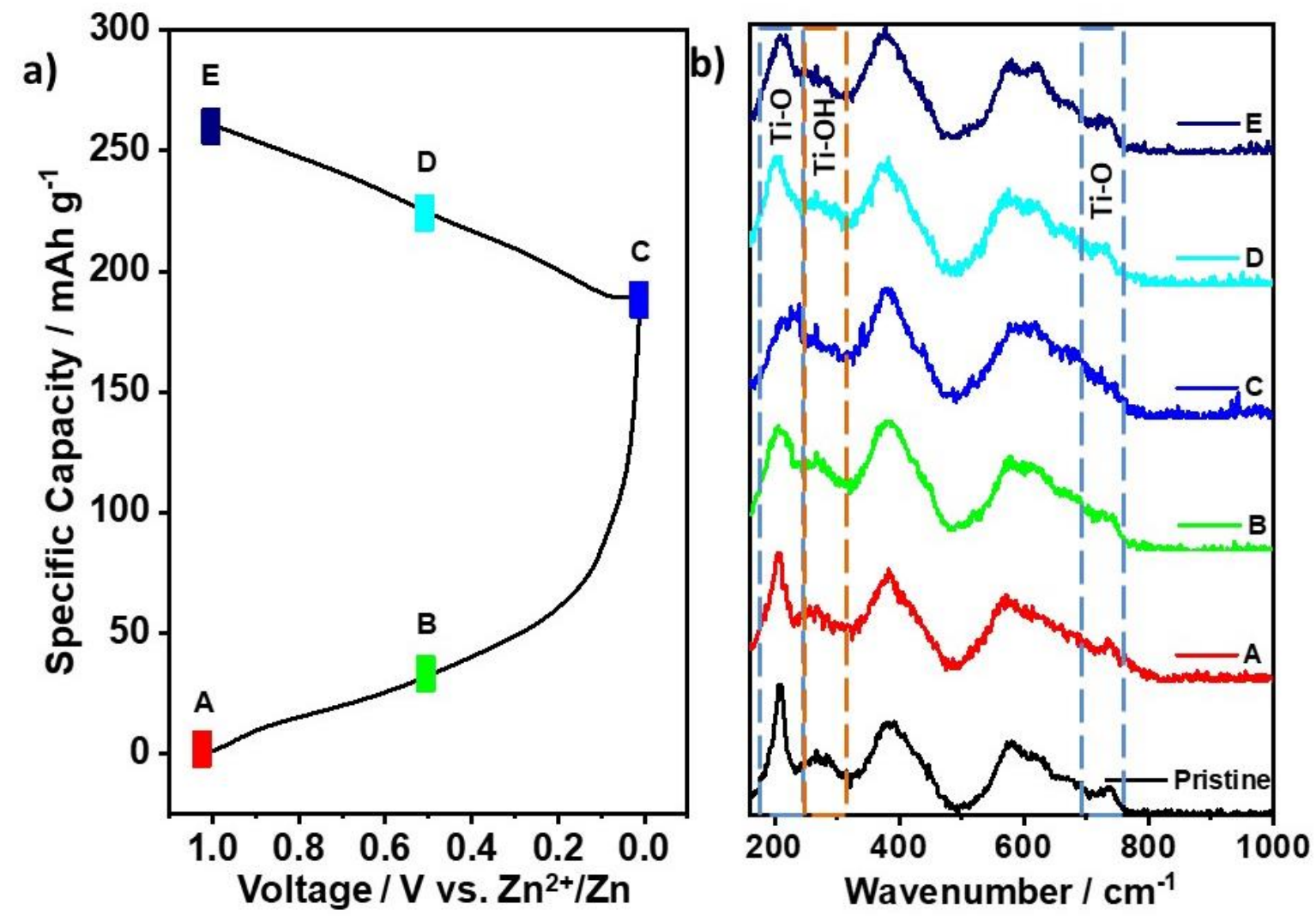

Figure 5. Ex-situ Raman spectroscopy data of the in-situ pillared $\mathrm{Ti}_{3} \mathrm{C}_{2}$ on the first cycle a) Load curve highlighting the state of charge each electrode was stopped at. b) Corresponding Raman spectra for each state of charge. The dashed rectangles highlight key $\mathrm{Ti}-\mathrm{O}(\mathrm{H})$ vibrations for the $\mathrm{Zn}$-intercalation mechanism.

The Raman spectrum is unchanged between the pristine electrode and the electrode at OCV even though the ex-situ EDS (Table S1) and XRD (Figure S8) data showed significant uptake of Zn ions into the interlayer. This shows that although $\mathrm{Zn}$ can spontaneously intercalate into $\mathrm{Ti}_{3} \mathrm{C}_{2}$ when the MXene is soaked in $\mathrm{ZnSO}_{4}$, no changes in the surface bonding occur, and the $\mathrm{Zn}$ has no covalent interaction with the termination groups.

In contrast, when current is drawn and cycling begins, there are several noticeable features in the voltage-dependent evolution of the peaks corresponding to the $-\mathrm{O}$ and $-\mathrm{OH}$ surface groups. During the first discharge cycle, the $\mathrm{Ti}=\mathrm{O}$ vibration at $210 \mathrm{~cm}^{-1}$ broadens and loses intensity. In addition, a shoulder develops at higher wavenumbers on the right-hand side of this peak. At $0.01 \mathrm{~V}$ this shoulder merges with the $270 \mathrm{~cm}^{-1} \mathrm{Ti}-\mathrm{O}-\mathrm{H}$ peak which also appears to broaden upon cycling. Both peaks are significantly flattened by the end of the first discharge and the $\mathrm{Ti}-\mathrm{O}-\mathrm{H}$ peak can no longer be distinguished. These changes then reverse on the first charging cycle, with the $210 \mathrm{~cm}^{-1}$ peak sharpening and seeing an increase in intensity and a reduction of the shoulder. By the end of the first charging cycle $(1 \mathrm{~V})$, the Ti-O-H peak at $270 \mathrm{~cm}^{-1}$ can be clearly distinguished from the $210 \mathrm{~cm}^{-1}$ shoulder once more. However, it should be noted that the Raman spectrum at the end of the first charging cycle is not the same as the pre-cycled electrode at OCV (also around $1 \mathrm{~V}$ ), suggesting that the changes in bonding are not fully reversible. In addition, EDS data showed significant amounts of $\mathrm{Zn}$ was retained in the material after charging to $1 \mathrm{~V}$ (Table S1). This explains the substantial irreversible capacity loss observed in the galvanostatic charge-discharge cycling (Figure 2); significant 
amounts of $\mathrm{Zn}$ are still bonded to the Ti-O groups by the end of the first charge to $1 \mathrm{~V}$. It is possible that further $\mathrm{Zn}$ could be extracted at higher voltages, however the $\mathrm{CV}$ analysis of the voltage window showed that the $\mathrm{Ti}_{3} \mathrm{C}_{2}$ caused significant water splitting above $1.2 \mathrm{~V}$, which limits the charging voltage.

These changes are consistent with $\mathrm{Zn}$ ions bonding and debonding to $\mathrm{Ti}=\mathrm{O}$ terminations on the $\mathrm{Ti}_{3} \mathrm{C}_{2}$ surface upon discharging and charging respectively. This is further supported by the broadening and sharpening of the Ti=O peak at $740 \mathrm{~cm}^{-1}$ upon cycling. The shoulder of the $210 \mathrm{~cm}^{-1}$ peak suggests the formation of a Ti-O-Zn type interaction, and occurs at lower wavenumbers than the Ti-O-H vibration due to the heavier mass of $\mathrm{Zn}$ compared to $\mathrm{H}$. This shows that $-\mathrm{O}$ terminations are the active sites for $\mathrm{Zn}$ storage in the MXene, which has also been reported for $\mathrm{H}^{+}$and $\mathrm{Na}^{+}$ion systems. ${ }^{45}$ This gives important guidance for the further improvement of capacity for $\mathrm{Ti}_{3} \mathrm{C}_{2}$ electrodes in $\mathrm{Zn}$ systems, since the number of $-\mathrm{O}$ terminal groups can easily be increased, for example by exchanging $-\mathrm{F}$ groups for O ones. ${ }^{57}$ Importantly, this is the first time MXenes have been shown to undergo faradaic processes in aqueous multivalent energy storage systems. Finally, we note that the addition of CTAB seems to have no effect on the underlying charge storage mechanism since the Raman spectra with and without CTAB show identical trends. This suggests that the improvements from the addition of CTAB are due to changes in the interlayer spacings and subsequent diffusion barriers rather than fundamentally changing the mechanisms involved.

These results strongly suggest that the dominant charge storage mechanism is from the intercalation of $\mathrm{Zn}$ between the $\mathrm{Ti}_{3} \mathrm{C}_{2}$ layers. We observe changes in intensity for the Ti-O peaks, showing that in $\mathrm{ZnSO}_{4}$ charge storage is predominantly through the reversible binding of $\mathrm{Zn}$ ions to the $-\mathrm{O}$ termination groups on the $\mathrm{Ti}_{3} \mathrm{C}_{2}$ surface. As was reported for $\mathrm{H}_{2} \mathrm{SO}_{4},{ }^{45}$ this must be accompanied by a change in the Ti valence state, i.e., a pseudocapacitive intercalation reaction. It is also noteworthy that the changes we report are not consistent with $\mathrm{H}^{+}$as the main charge carrier, since we do not observe the distinctive shift in the Ti=O peaks. In fact, when the cell is fully discharged, and the Ti-O-H groups would be at their most abundant if $\mathrm{H}^{+}$was the charge carrier, the Ti-O-H peak at $270 \mathrm{~cm}^{-1}$ cannot be distinguished in our Raman spectra. In addition, $0.1 \mathrm{M} \mathrm{ZnSO}_{4}$ contains a low concentration of $\mathrm{H}^{+}(\mathrm{pH} 4-6)$, which are insufficient to account for the capacities reported here. ${ }^{45}$ Finally, since no $\mathrm{S}$ is detected in our EDS results (Table S1) and there is no $\mathrm{SO}_{4}{ }^{2-}$ peak $\left(980 \mathrm{~cm}^{-1}\right)^{44}$ in our Raman spectra (Figure 5), electric double layer capacitance via $\mathrm{SO}_{4}{ }^{2-}$ adsorption can also be ruled out as the charge storage mechanism. Therefore, the results are strongly consistent with the reversible storage of $\mathrm{Zn}^{2+}$ ions as the main charge carrier.

Ex-situ XPS was then used on the in-situ pillared $\mathrm{Ti}_{3} \mathrm{C}_{2}$ to investigate the $\mathrm{Ti}$ oxidation state during cycling. The results are shown in Figure S9. There are four broad main peaks that can be observed in the Ti $2 p$ spectra for the OCV sample (Figure S9, A). These centre on 455.9, 459.7, 462.0 and $465.2 \mathrm{eV}$. This is consistent with XPS spectra from previously reported studies, which show that $\mathrm{Ti}_{3} \mathrm{C}_{2}$ contains predominately $\mathrm{Ti}^{3+}$ and $\mathrm{Ti}^{2+} .62,63 \mathrm{Ti}^{4+}$ oxidation states correspond to surface oxides from the oxidation of the MXene, while the other states are all expected to be found in MXenes.

Upon discharge, there is a clear shift in all peaks to lower binding energies, which corresponds to the presence of Ti species with lower oxidation states. For example, the peak at $455.9 \mathrm{eV}$ (3/2 electrons) at OCV shifts to $455.4 \mathrm{eV}$ at $0.01 \mathrm{~V}$, implying reduction of $\mathrm{Ti}^{2+}$ to $\mathrm{Ti}^{0}$. A similar shift is observed for the $462.0 \mathrm{eV}$ peak (1/2 electrons), which occurs at $461.5 \mathrm{eV}$ at $0.01 \mathrm{~V}$, further supporting the MXene Ti reduction. At $0.5 \mathrm{~V}$, the corresponding peaks sit between these two end points, at $455.6 \mathrm{eV}$ for the 
$3 / 2$ electrons and $461.4 \mathrm{eV}$ for the $1 / 2$ electrons, suggesting that the $\mathrm{Ti}$ is reduced throughout the voltage window. This implies that at voltages between 0.5 and $1 \mathrm{~V}$ the charge storage has significant contribution from pseudocapacitive mechanisms, not just EDL capacitance (this matches data from CV plots Figure 3 ). When the cell is charged, these changes reverse, showing that the change in valence state upon discharge is mostly reversible, revealing that the $\mathrm{Zn}$ storage is achieved via a Ti-based redox reaction. Interestingly, the electrode charged to $0.5 \mathrm{~V}$ shows very similar shifts to the electrode charged to $1 \mathrm{~V}(0.1 \mathrm{eV}$ and $0.2 \mathrm{eV}$ lower binding energies than at $1 \mathrm{~V}$ for the $3 / 2$ and $1 / 2$ electrons respectively). This implies that on charge most of the redox contribution has occurred by $0.5 \mathrm{~V}$, which would match the distinctive redox peak observed on the CV (Figure 3). Reversible Ti redox is also shown by the peak deconvolution, where the concentration of $\mathrm{Ti}^{2+}$ increases upon discharge (from $28 \%$ at OCV to $34 \%$ at $0.01 \mathrm{~V}$ ) and decreases on the subsequent charge (to $30 \%$ ), supporting the reversible reduction of $\mathrm{Ti}^{4+}$ and $\mathrm{Ti}^{3+}$ to $\mathrm{Ti}^{2+}$. This behaviour has previously been demonstrated for $\mathrm{Ti}_{3} \mathrm{C}_{2}$ MXene using in-situ XANES experiments in aqueous $\mathrm{H}_{2} \mathrm{SO}_{4}$ and organic Li-ion systems. ${ }^{64,65}$ In both these cases the overall titanium oxidation state in the MXene was just over $2^{+}$, which would seem to match well with our results for $\mathrm{Zn}$.

Furthermore, XPS data show that the surface oxides $\left(\mathrm{Ti}^{4+}\right)$ also change upon cycling. As well as a reversible shift on charge and discharge as seen for the MXene $\mathrm{Ti}$, there is also a broadening on discharge (to the extent that the $1 / 2 \mathrm{Ti}^{4+}$ component at $456.3 \mathrm{eV}$ cannot be distinguished at $0.01 \mathrm{~V}$ and the $3 / 2$ component at $459.5 \mathrm{eV}$ is severely flattened). The concentration of $\mathrm{Ti}^{4+}$ in the spectra decreases from $22 \%$ to $15 \%$ between OCV and $0.01 \mathrm{~V}$, further supporting the $\mathrm{Ti}^{4+}$ reduction. This is then reversed on charging to $0.5 \mathrm{~V}$, with little difference between the 0.5 and $1 \mathrm{~V}$ electrodes. This suggests that the surface oxides also contribute to reversible $\mathrm{Zn}$ storage, and that the oxidation on charge occurs almost entirely between 0.01 and $0.5 \mathrm{~V}$. However, it may be that these surface oxides are still not desirable for MXene performance, since a previous study on nanosized anatase $\mathrm{TiO}_{2}$ for $\mathrm{Zn}$-ion batteries reported low capacities of $25 \mathrm{mAh} \mathrm{g}{ }^{-1}$, much less than $\mathrm{Ti}_{3} \mathrm{C}_{2}$ reported here. ${ }^{66}$ Further work on the role of titanium-based oxides in $\mathrm{Zn}$-ion systems is needed to fully determine their performance and contribution here.

The mechanistic understanding yielded by our results allows for the optimisation and improvement of the MXene materials as $\mathrm{Zn}$-ion hosts. This is demonstrated by the cycling results in Figure 6 , which were carried out in an elevated voltage window of 0.2-1.2 $\mathrm{V}$, thus removing the diffusion-limited processes that the $\mathrm{CV}$ analysis showed were below $0.2 \mathrm{~V}$. In this voltage window, we were able to observe increased coulombic efficiency ( $99.5 \%$ compared to $39 \%$ in the $0.01-1.0 \mathrm{~V}$ window) signifying enhanced $\mathrm{Zn}$ storage reversibility resulting from the capacitive processes. This is much closer to levels required for practical applications, although this occurs at the expense of delivering lower initial specific capacities (73 $\mathrm{mAh} \mathrm{g}^{-1}$ compared to $189 \mathrm{mAh} \mathrm{g}^{-1}$ in the $0.01-1.0 \mathrm{~V}$ window). Nevertheless, the impressive coulombic efficiency was maintained above $99 \%$ across 100 cycles at $20 \mathrm{~mA} \mathrm{~g}^{-1}$. This was coupled with outstanding cycling stability, with over $96 \%$ capacity retention between the 1 st and 100th cycles. This compared with $56 \%$ retention for the non-pillared MXene (which showed irreversible plateaus at voltages above $1.1 \mathrm{~V}$, Figure S10) showing that the non-pillared material was less stable in this higher voltage window, further demonstrating the benefits of our unique in-situ pillaring method. Both the pillared and non-pillared materials show coulombic efficiencies slightly over $100 \%$ in initial cycles. This is explained by the extraction of the spontaneously intercalated $\mathrm{Zn}$ which our ex-situ EDS (Table S1) and XRD results (Figure 4 and S6) revealed are present in the MXene at OCV, which are being deintercalated at these elevated voltages. Pre-intercalation of the charge carrying ion has been observed to lead to initial coulombic efficiencies over $100 \%$ in other systems, including aqueous $\mathrm{Zn}$-ion systems. ${ }^{67-69}$ Alternatively, there could be side-reactions between the electrode and electrolyte in the high voltage region in these initial cycles. ${ }^{67,70,71}$ Importantly, our system rapidly 
stabilises to coulombic efficiencies just under $100 \%$, showing that the system quickly stabilises enabling efficient and long-term cycling. Even at high rates, the in-situ pillared MXene showed excellent capacity retention with over $96 \%$ of the initial capacity retained even after 1,000 cycles at the elevated rate of $0.2 \mathrm{~A} \mathrm{~g}^{-1}$ (Figure 6c), with coulombic efficiencies around $100 \%$. These results demonstrate that pillared $\mathrm{Ti}_{3} \mathrm{C}_{2} \mathrm{MXene}$ is a promising material for long-life aqueous $\mathrm{Zn}$-ion systems.

a)

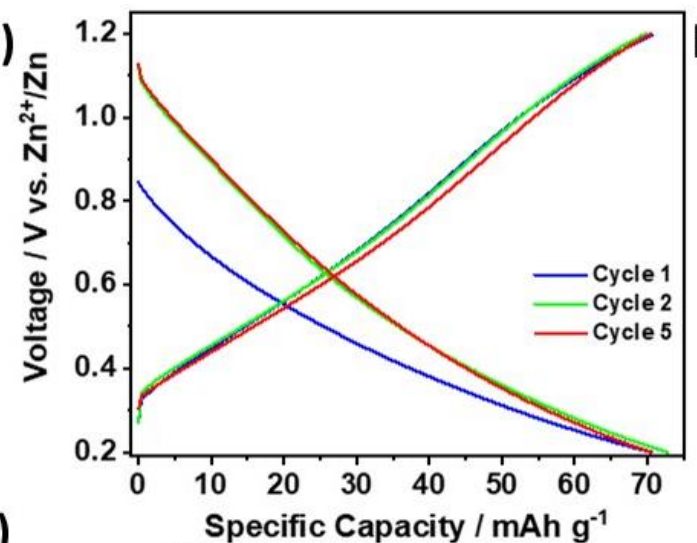

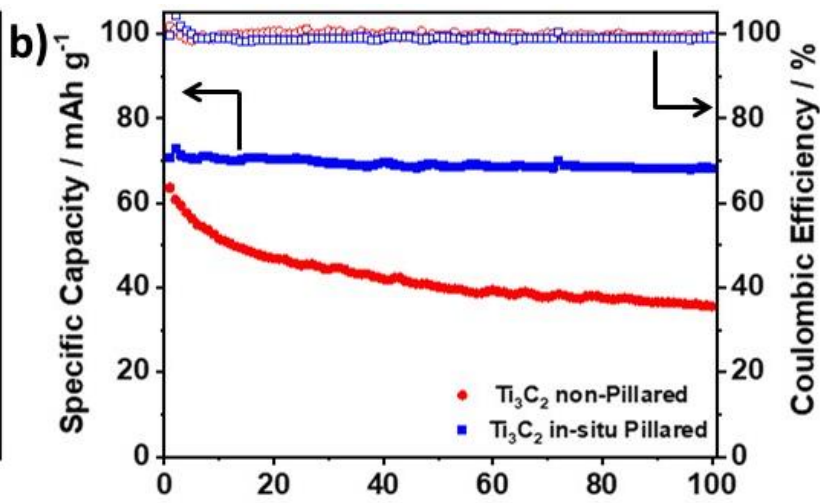

Cycle Number

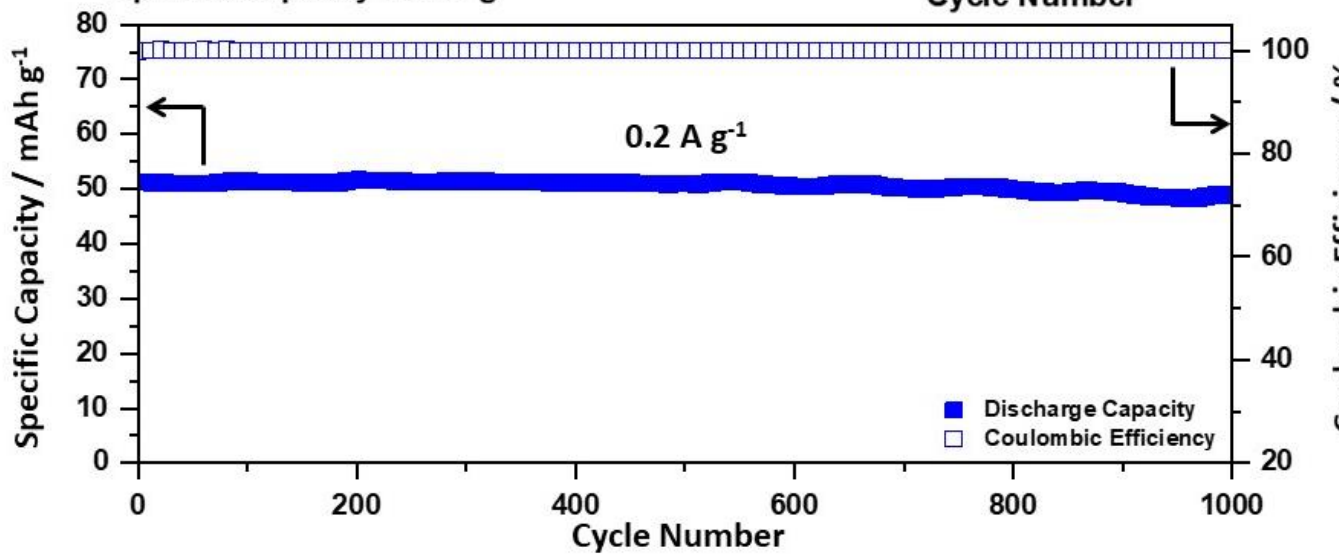

Figure 6. a) Load curves in the voltage window of $0.2-1.2 \mathrm{~V} \mathrm{vs} . \mathrm{Zn}^{2+} / \mathrm{Zn}$ at a current density of $20 \mathrm{~mA} \mathrm{~g}^{-}$ ${ }^{1}$ for the in-situ pillared $\mathrm{Ti}_{3} \mathrm{C}_{2}$. (b) Discharge capacities and Coulombic efficiencies over 100 cycles at a rate of $20 \mathrm{~mA} \mathrm{~g}^{-1}$ across a voltage window of 0.2-1.2 $\mathrm{V}$ for in-situ pillared (blue) and non-pillared (red) $\mathrm{Ti}_{3} \mathrm{C}_{2}$. c) Long-term cycling stability test of the in-situ pillared $\mathrm{Ti}_{3} \mathrm{C}_{2}$ at an elevated rate of $0.2 \mathrm{~A} \mathrm{~g}^{-1}$ in the voltage range of $0.2-1.2 \mathrm{~V}$ over 1,000 cycles.

\section{Conclusions}

In summary, in this work, we demonstrate the viability of $\mathrm{Ti}_{3} \mathrm{C}_{2} \mathrm{MXene}$ as a zinc-ion host for future sustainable zinc-ion hybrid capacitors. Furthermore, we have presented a proof-of-concept structuring technique using surfactant additives to control the electrode architecture via a novel insitu pillaring method. The insights gained from kinetic, diffraction and spectroscopic studies reveal that the $\mathrm{Zn}^{2+}$ (de)intercalation occurs via pseudocapacitive and battery-like processes, with the -0 functional groups playing a key role as the binding site for $\mathrm{Zn}$ ions. This is the first time MXenes have been shown to store charge via faradaic processes in aqueous multivalent energy storage systems. The in-situ pillaring both expands the MXene layers, which promote the pseudocapacitive properties of the material and evade volume changes during cycling. In particular, this resulted in outstanding cycling stability, with over $96 \%$ capacity retention achieved even after 1,000 cycles. This work opens new opportunities for using in-situ structural approaches to improve electrode performance, which in 
turn, would significantly simplify the use of 2D materials as electrode materials, where complicated processes are often used to control the structure. The in-situ pillaring could be applied not just to other MXenes, but also to the wider group of 2D and layered materials. Finally, we note that MXenes are a growing family of over $\mathbf{2 0}$ members, and that work on MXenes in other ion systems has seen substantial improvements in performance via control of the flake size and surface groups combined with careful control of the electrode architecture, which demonstrates the large potential MXenes can be expected to have in zinc-ion systems.

\section{References}

1. Lu, X. \& Yang, Z. Advances in Batteries for Medium and Large-Scale Energy Storage. Advances in Batteries for Medium and Large-Scale Energy Storage (Elsevier, 2015). doi:10.1016/B978-178242-013-2.00005-4.

2. Dunn, B., Kamath, H. \& Tarascon, J.-M. Electrical energy storage for the grid: a battery of choices. Science 334, 928-35 (2011).

3. Ottakam Thotiyl, M. M. et al. A stable cathode for the aprotic Li-O2 battery. Nat. Mater. 12, 1050-6 (2013).

4. Grosjean, C., Miranda, P. H., Perrin, M. \& Poggi, P. Assessment of world lithium resources and consequences of their geographic distribution on the expected development of the electric vehicle industry. Renew. Sustain. Energy Rev. 16, 1735-1744 (2012).

5. Mauger, A. \& Julien, C. M. Critical review on lithium-ion batteries: are they safe? Sustainable? lonics (Kiel). 23, 1933-1947 (2017).

6. Song, M., Tan, H., Chao, D. \& Fan, H. J. Recent Advances in Zn-lon Batteries. Adv. Funct. Mater. 28, 1802564 (2018).

7. Fang, G., Zhou, J., Pan, A. \& Liang, S. Recent Advances in Aqueous Zinc-lon Batteries. ACS Energy Lett. 3, 2480-2501 (2018).

8. Wei, T., Li, Q., Yang, G. \& Wang, C. Highly reversible and long-life cycling aqueous zinc-ion battery based on ultrathin $\left(\mathrm{NH}_{4}\right)_{2} \mathrm{~V}_{10} \mathrm{O}_{25} \cdot 8 \mathrm{H}_{2} \mathrm{O}$ nanobelts. J. Mater. Chem. A 6, 2040220410 (2018).

9. Kundu, D., Adams, B. D., Duffort, V., Vajargah, S. H. \& Nazar, L. F. A high-capacity and long-life aqueous rechargeable zinc battery using a metal oxide intercalation cathode. Nat. Energy 1 , 16119 (2016).

10. Shen, C. et al. Graphene-Boosted, High-Performance Aqueous Zn-Ion Battery. ACS Appl. Mater. Interfaces 10, 25446-25453 (2018).

11. Suo, L. et al. 'Water-in-salt' electrolyte enables high-voltage aqueous lithium-ion chemistries. Science 350, 938-43 (2015).

12. Kim, H. et al. Aqueous Rechargeable Li and Na Ion Batteries. Chem. Rev. 114, 11788-11827 (2014).

13. Alfaruqi, M. H. et al. Electrochemically Induced Structural Transformation in a $\mathrm{y}-\mathrm{MnO}_{2}$ Cathode of a High Capacity Zinc-Ion Battery System. Chem. Mater. 27, 3609-3620 (2015).

14. Senguttuvan, P. et al. A High Power Rechargeable Nonaqueous Multivalent $\mathrm{Zn} / \mathrm{V}_{2} \mathrm{O}_{5}$ Battery. Adv. Energy Mater. 6, 1600826 (2016).

15. Alfaruqi, M. H. et al. A layered $\delta-\mathrm{MnO} 2$ nanoflake cathode with high zinc-storage capacities for eco-friendly battery applications. Electrochem. commun. 60, 121-125 (2015).

16. Lee, J., Ju, J. B., Cho, W. II, Cho, B. W. \& Oh, S. H. Todorokite-type MnO2 as a zinc-ion intercalating material. Electrochim. Acta 112, 138-143 (2013).

17. Alfaruqi, M. H. et al. Electrochemically Induced Structural Transformation in a $\mathrm{\gamma}-\mathrm{MnO}_{2}$ Cathode of a High Capacity Zinc-Ion Battery System. Chem. Mater. 27, 3609-3620 (2015).

18. Xia, C. et al. Rechargeable Aqueous Zinc-Ion Battery Based on Porous Framework Zinc Pyrovanadate Intercalation Cathode. Adv. Mater. 30, 1705580 (2018). 
19. Hu, P. et al. Zn/ $\mathrm{V}_{2} \mathrm{O}_{5}$ Aqueous Hybrid-Ion Battery with High Voltage Platform and Long Cycle Life. ACS Appl. Mater. Interfaces 9, 42717-42722 (2017).

20. Sambandam, B. et al. Aqueous rechargeable $\mathrm{Zn}$-ion batteries: an imperishable and highenergy $\mathrm{Zn}_{2} \mathrm{~V}_{2} \mathrm{O}_{7}$ nanowire cathode through intercalation regulation. J. Mater. Chem. A 6, 3850-3856 (2018).

21. Zhang, L., Chen, L., Zhou, X. \& Liu, Z. Towards High-Voltage Aqueous Metal-lon Batteries Beyond 1.5 V: The Zinc/Zinc Hexacyanoferrate System. Adv. Energy Mater. 5, 1400930 (2015).

22. Jia, Z., Wang, B. \& Wang, Y. Copper hexacyanoferrate with a well-defined open framework as a positive electrode for aqueous zinc ion batteries. Mater. Chem. Phys. 149-150, 601-606 (2015).

23. Chae, M. S., Heo, J. W., Kwak, H. H., Lee, H. \& Hong, S.-T. Organic electrolyte-based rechargeable zinc-ion batteries using potassium nickel hexacyanoferrate as a cathode material. J. Power Sources 337, 204-211 (2017).

24. Raccichini, R., Varzi, A., Passerini, S. \& Scrosati, B. The role of graphene for electrochemical energy storage. Nat. Mater. 14, 271-9 (2015).

25. Kucinskis, G., Bajars, G. \& Kleperis, J. Graphene in lithium ion battery cathode materials: A review. J. Power Sources 240, 66-79 (2013).

26. Ouyang, B., Wang, Y., Zhang, Z. \& Rawat, R. S. MoS2 anchored free-standing three dimensional vertical graphene foam based binder-free electrodes for enhanced lithium-ion storage. Electrochim. Acta 194, 151-160 (2016).

27. Park, J. et al. Discharge mechanism of MoS2 for sodium ion battery: Electrochemical measurements and characterization. Electrochim. Acta 92, 427-432 (2013).

28. He, P. et al. Layered VS 2 Nanosheet-Based Aqueous Zn Ion Battery Cathode. Adv. Energy Mater. 7, 1601920 (2017).

29. Dai, X., Wan, F., Zhang, L., Cao, H. \& Niu, Z. Freestanding graphene/VO2 composite films for highly stable aqueous $\mathrm{Zn}$-ion batteries with superior rate performance. Energy Storage Mater. 17, 143-150 (2019).

30. Naguib, M. et al. Two-dimensional nanocrystals produced by exfoliation of Ti3 AIC2. Adv. Mater. 23, 4248-53 (2011).

31. Ghidiu, M. et al. Synthesis and characterization of two-dimensional Nb4C3 (MXene). Chem. Commun. (Camb). 50, 9517-20 (2014).

32. Naguib, M. et al. Two-dimensional transition metal carbides. ACS Nano 6, 1322-31 (2012).

33. Naguib, M., Mochalin, V. N., Barsoum, M. W. \& Gogotsi, Y. 25th Anniversary Article: MXenes: A New Family of Two-Dimensional Materials. Adv. Mater. 26, 992-1005 (2014).

34. Sun, D. et al. Two-dimensional Ti3C2 as anode material for Li-ion batteries. Electrochem. commun. 47, 80-83 (2014).

35. Zhu, J., Chroneos, A. \& Schwingenschlögl, U. Nb-based MXenes for Li-ion battery applications. Phys. status solidi - Rapid Res. Lett. 9, 726-729 (2015).

36. Mashtalir, O., Lukatskaya, M. R., Zhao, M.-Q., Barsoum, M. W. \& Gogotsi, Y. Amine-Assisted Delamination of Nb2C MXene for Li-lon Energy Storage Devices. Adv. Mater. 27, 3501-6 (2015).

37. Kajiyama, S. et al. Sodium-Ion Intercalation Mechanism in MXene Nanosheets. ACS Nano 10, 3334-3341 (2016).

38. Dall'Agnese, Y., Taberna, P.-L., Gogotsi, Y. \& Simon, P. Two-Dimensional Vanadium Carbide (MXene) as Positive Electrode for Sodium-Ion Capacitors. J. Phys. Chem. Lett. 6, 2305-2309 (2015).

39. Wang, X. et al. Pseudocapacitance of MXene nanosheets for high-power sodium-ion hybrid capacitors. Nat. Commun. 6, 6544 (2015).

40. Xu, M. et al. Opening Magnesium Storage Capability of Two-Dimensional MXene by Intercalation of Cationic Surfactant. ACS Nano 12, 3733-3740 (2018).

41. Yu, X. et al. Mg intercalation into Ti2C building block. Chem. Phys. Lett. 629, 36-39 (2015). 
42. VahidMohammadi, A., Hadjikhani, A., Shahbazmohamadi, S. \& Beidaghi, M. Two-Dimensional Vanadium Carbide (MXene) as a High-Capacity Cathode Material for Rechargeable Aluminum Batteries. ACS Nano 11, 11135-11144 (2017).

43. Levi, M. D. et al. Solving the Capacitive Paradox of 2D MXene using Electrochemical QuartzCrystal Admittance and In Situ Electronic Conductance Measurements. Adv. Energy Mater. 5, n/a-n/a (2015).

44. Ghidiu, M., Lukatskaya, M. R., Zhao, M.-Q., Gogotsi, Y. \& Barsoum, M. W. Conductive twodimensional titanium carbide 'clay' with high volumetric capacitance. Nature 516, 78-81 (2014).

45. Hu, M. et al. High-Capacitance Mechanism for $\mathrm{Ti}_{3} \mathrm{C}_{2} T_{\times}$MXene by in Situ Electrochemical Raman Spectroscopy Investigation. ACS Nano 10, 11344-11350 (2016).

46. Yang, Q. et al. A Wholly Degradable, Rechargeable $\mathrm{Zn}-\mathrm{Ti}_{3} \mathrm{C}_{2} \mathrm{MXene}$ Capacitor with Superior Anti-Self-Discharge Function. ACS Nano acsnano.9b03650 (2019) doi:10.1021/acsnano.9b03650.

47. Wang, Q. et al. MXene-Reduced Graphene Oxide Aerogel for Aqueous Zinc-lon Hybrid Supercapacitor with Ultralong Cycle Life. Adv. Electron. Mater. 1900537 (2019) doi:10.1002/aelm.201900537.

48. Anasori, B., Lukatskaya, M. R. \& Gogotsi, Y. 2D metal carbides and nitrides (MXenes) for energy storage. Nat. Rev. Mater. 2, 16098 (2017).

49. Yan, J. et al. Flexible MXene/Graphene Films for Ultrafast Supercapacitors with Outstanding Volumetric Capacitance. Adv. Funct. Mater. 27, 1701264 (2017).

50. Tian, Y. et al. Surface nitrogen-modified 2D titanium carbide (MXene) with high energy density for aqueous supercapacitor applications. J. Mater. Chem. A 7, 5416-5425 (2019).

51. Zhang, C. J. et al. Oxidation Stability of Colloidal Two-Dimensional Titanium Carbides (MXenes). Chem. Mater. 29, 4848-4856 (2017).

52. Luo, J. et al. Pillared Structure Design of MXene with Ultralarge Interlayer Spacing for HighPerformance Lithium-Ion Capacitors. ACS Nano 11, 2459-2469 (2017).

53. Cheng, R. et al. Understanding the Lithium Storage Mechanism of Ti3C2Tx MXene. J. Phys. Chem. C 123, 1099-1109 (2019).

54. Xie, X. et al. Porous Heterostructured MXene/Carbon Nanotube Composite Paper with High Volumetric Capacity for Sodium-Based Energy Storage Devices. Nano Energy (2016) doi:10.1016/j.nanoen.2016.06.005.

55. Lukatskaya, M. R. et al. Cation intercalation and high volumetric capacitance of twodimensional titanium carbide. Science 341, 1502-5 (2013).

56. Kong, L. et al. Ultrahigh intercalation pseudocapacitance of mesoporous orthorhombic niobium pentoxide from a novel cellulose nanocrystal template. Mater. Chem. Phys. 149, 495-504 (2015).

57. Augustyn, V. et al. High-rate electrochemical energy storage through Li+ intercalation pseudocapacitance. Nat. Mater. 12, 518-522 (2013).

58. Yoder, C. H. Ionic compounds : applications of chemistry to mineralogy. (Wiley-Interscience, 2006).

59. Cauët, E. et al. Structure and dynamics of the hydration shells of the $\mathrm{Zn} 2+$ ion from ab initio molecular dynamics and combined $a b$ initio and classical molecular dynamics simulations. $J$. Chem. Phys. 132, 194502 (2010).

60. Wang, X. et al. Pseudocapacitance of MXene nanosheets for high-power sodium-ion hybrid capacitors. Nat. Commun. 6, 6544 (2015).

61. Hu, $\mathrm{T}$. et al. Vibrational properties of $\mathrm{Ti}_{3} \mathrm{C}_{2}$ and $\mathrm{Ti}_{3} \mathrm{C}_{2} \mathrm{~T}_{2}(\mathrm{~T}=\mathrm{O}, \mathrm{F}, \mathrm{OH})$ monosheets by firstprinciples calculations: a comparative study. Phys. Chem. Chem. Phys. 17, 9997-10003 (2015).

62. Halim, J. et al. X-ray photoelectron spectroscopy of select multi-layered transition metal carbides (MXenes). Appl. Surf. Sci. 362, 406-417 (2016).

63. Luo, J. et al. Tunable pseudocapacitance storage of MXene by cation pillaring for high 
performance sodium-ion capacitors. J. Mater. Chem. A 6, 7794-7806 (2018).

64. Lukatskaya, M. R. et al. Probing the Mechanism of High Capacitance in 2D Titanium Carbide Using In Situ X-Ray Absorption Spectroscopy. Adv. Energy Mater. 5, 1500589 (2015).

65. Xie, Y. et al. Role of surface structure on Li-ion energy storage capacity of two-dimensional transition-metal carbides. J. Am. Chem. Soc. 136, 6385-94 (2014).

66. Liu, W. et al. Investigation of zinc ion storage of transition metal oxides, sulfides, and borides in zinc ion battery systems. Chem. Commun. 53, 6872-6874 (2017).

67. Khamsanga, S., Pornprasertsuk, R., Yonezawa, T., Mohamad, A. A. \& Kheawhom, S. ס-MnO2 nanoflower/graphite cathode for rechargeable aqueous zinc ion batteries. Sci. Rep. 9, 1-9 (2019).

68. Zhu, B., Wang, X., Yao, P., Li, J. \& Zhu, J. Towards high energy density lithium battery anodes: Silicon and lithium. Chemical Science vol. 10 7132-7148 (2019).

69. Wu, D. et al. NaV6015: a promising cathode material for insertion/extraction of $\mathrm{Mg} 2+$ with excellent cycling performance. Nano Res. 1-9 (2020) doi:10.1007/s12274-019-2602-6.

70. Wang, S., Kravchyk, K. V., Krumeich, F. \& Kovalenko, M. V. Kish Graphite Flakes as a Cathode Material for an Aluminum Chloride-Graphite Battery. ACS Appl. Mater. Interfaces 9, 2847828485 (2017).

71. Angell, M. et al. High Coulombic efficiency aluminum-ion battery using an $\mathrm{AlCl} 3$-urea ionic liquid analog electrolyte. Proc. Natl. Acad. Sci. U. S. A. 114, 834-839 (2017).

\section{Acknowledgements}

PAM would like to acknowledge funding from the EPSRC via the Graphene NOWNANO Centre for Doctoral Training. NTR would like to acknowledge Royal Society (RG170150) for provision of funds to conduct this research. NB would like to acknowledge Lancaster University for an Early Career Internal Grant. XPS data collection was performed at the EPSRC National Facility for XPS ('HarwellXPS'), operated by Cardiff University and UCL, under contract No. PR16195. 